\title{
THE HYPEROSCULATING SPACES TO CERTAIN CURVES IN $[n]$
}

\author{
by R. H. DYE \\ (Received 5th February 1974)
}

\section{Introduction}

Let $\Gamma$ be an irreducible and non-singular curve in $[n](n \geqq 3)$ which is the complete intersection of $n-1$ primals of order $m(m \geqq 2)$ with a common " selfpolar" simplex $S$ : by this I mean that the $r$ th polar of each vertex of $S$ with respect to any one of the defining primals is the opposite face of $S$ counted $m-r$ times, for $r=1,2, \ldots, m-1$. The various such $\Gamma$ constitute the curves of the title; they were encountered in (2). When $m=2, \Gamma$ is the intersection of $n-1$ quadrics with a common self-polar simplex in the familiar classical sense.

A special feature of $\Gamma$, and one of its claims to interest, is the exceptionally high order of contact of the osculating spaces at the points of hyperosculation: points where the osculating prime has $(n+1)$-point contact (intersection) or more. The points of hyperosculation on $\Gamma$ are its intersections with the faces of $S$; the osculating [s] at these points have $m s$-point contact and so are hyperosculating (except when $s=1$ and $m=2$ ). All the other points on $\Gamma$ are ordinary: their osculating $[s]$ have the statutory minimal $(s+1)$-point contact. On a general curve in $[n]$ where, in contrast to $\Gamma$, there is no confluence of the points of hyperosculation, the hyperosculating primes have precisely $(n+1)$-point contact; while at the points of hyperosculation the osculating [s] for $s \leqq n-2$ are not hyperosculating. The facts about $\Gamma$ were established in $(2, \mathrm{pp} .334,335)$ by a somewhat intricate discussion. It involved limiting position arguments for osculating spaces-so our geometry was complex-a calculation of the genus of $\Gamma$, an evaluation of the multiplicities of the points of hyperosculation, and the use of a formula for their number, which in turn depends on correspondence theory.

A major concern of (2) was to find the equation of the osculating prime at an ordinary point on $\Gamma$. The discussion of $(2, \mathrm{pp} .335,336)$ for obtaining this equation is not valid at the points of hyperosculation. For one thing, equation (41) of (2) is invalid since a denominator vanishes at these points; another, more serious, reason is explained below. However, the equation of the osculating prime at a point of hyperosculation has the same form as that at an ordinary point, and one of our purposes here is to establish this. Our simple and uniform treatment gives the osculating $[n-1]$ at all points of $\Gamma$; and simultaneously exhibits the nature of the osculating $[s]$ at each point, and thus in particular, of the hyperosculating spaces. Only unsophisticated algebra is required, so our 
geometry is now, as it should be, over any algebraically closed field with characteristic zero. Being direct, unlike the a posteriori verification of (2), our present method yields a bonus: it gives equations for each osculating [s].

Our approach is by examination of the places or branches of $\Gamma$. At a simple point $P$ of a curve in $[n]$ there is a single linear place $(3$, p. 193). Provided the curve does not lie in a prime, coordinates $y=\left(y_{0}, y_{1}, \ldots, y_{n}\right)$ may be chosen so that a minimal parametrisation of the place in an indeterminate $t$ has the form

$$
y_{i}=b_{i} t^{t_{i}}+\text { higher powers of } t ; i=0,1, \ldots, n \text {, }
$$

where $b_{i} \neq 0 ; l_{0}=0, l_{1}=1$ and $2 \leqq l_{2}<l_{3}<\ldots<l_{n}$. The centre " $t=0$ " of the place is $P$, and the osculating [s] to the curve at $P$ is

$$
y_{s+1}=y_{s+2}=\ldots=y_{n}=0
$$

with $l_{s+1}$-point contact $(1$, Vol. 5, p. 199), (3, pp. 182, 183). We obtain explicitly each place of $\Gamma$ in the given coordinate system, find new coordinates so that the place is in canonical form, and then translate back. The canonical form for a place leads us to the second reason for the failure at the points of hyperosculation of $\Gamma$ of the method of (2) for obtaining the equations of osculating primes. Let $\xi, d \xi, d^{2} \xi, \ldots$ be the values of $y, d y, d^{2} y, \ldots$ at the centre $P(t=0)$ of a place, taken as above, of a curve. If the osculating [n-2] at $P$ is hyperosculating then $l_{n-1} \geqq n$. On writing down the $\xi, d \xi, \ldots, d^{n-1} \xi$ explicitly one sees immediately that they all lie in the $[n-2]: y_{n-1}=y_{n}=0$, and so do not span the osculating prime. On the other hand, if the osculating $[n-2]$ is ordinary then $l_{n-1}=n-1$. Hence $l_{i}=i$ for $i<n$, and so, whatever the value of $l_{n}, \xi, d \xi, \ldots, d^{n-1} \xi$ are linearly independent and do span the osculating prime. This fact for the ordinary points of $\Gamma$ was crucial in the proof of $(2, p .336)$.

The equation for the osculating prime at a point on the curve of intersection of $n-1$ general primals in $[n]$ only appears in the literature for $n=3$ and $n=4$, and then only for simple points whose osculating $[n-2]$ are not hyperosculating. So it is interesting to have the equation at all points on special curves in $[n]$. The general case for $n=4$ was discussed in (2, p. 331); the equation becomes vacuous at points with hyperosculating planes. Indeed, this was explicitly noticed on page 333 for the points of hyperosculation of the $\Gamma$ with $m=2$ and $n=4$. The general case for $n=3$ is classical. Careful writers, for example (1, Vol. 5, p. 206), observe that they are obtaining the equation at ordinary points; it is implicit in Baker's discussion (1, Vol. 5, pp. 206, 207, 208) that the equation is vacuous at inflexions, and this can quickly be verified explicitly. When $m=2$ and $n=3, \Gamma$ is the famous elliptic quartic which has no inflexions; the equations of the osculating planes at all points, obtained from the general result for $n=3$, have been known and used for more than a century. We refer the reader to the bibliography of (2) for a detailed list of appearances and properties of the $\Gamma$ with $m=2$ and $n \leqq 5$.

In the final paragraphs of this note we make some mention of the group of $\Gamma$ and its relevance to osculating and other tangential matters. 


\section{The places on $\boldsymbol{\Gamma}$}

2.1. Suppose that coordinates $x^{\prime}=\left(x_{0}, x_{1}, \ldots, x_{n}\right)$ are taken so that $S$ is the simplex of reference with vertices $X_{0}, X_{1}, \ldots, X_{n}$. Summations will always run over $0,1, \ldots, n$ unless the contrary is explicitly indicated. $\Gamma$ may be taken as

$$
\sum_{i} a_{i}^{j} x_{i}^{m}=0, j=0,1, \ldots, n-2,
$$

where the $a_{i}$ are distinct. This canonical form for $\Gamma$ is established by the discussion of $(2$, p. 334$)$ which, involving only simple geometry and algebra, may be presumed here. The primals appearing in (1) are linear combinations of the original $m$-ics defining $\Gamma$. If $\lambda$ is fixed then we may replace each $a_{i}$ by $a_{i}+\lambda$, since this is equivalent to taking $n-1$ independent linear combinations of the primals in (1). Hence we may take all the $a_{i}$ to be non-zero, and then the equations of (1) represent non-singular $m$-ics.

2.2. We define

where dashes here denote derivatives. Then

$$
f(\theta)=\left(\theta-a_{0}\right)\left(\theta-a_{1}\right) \ldots\left(\theta-a_{n}\right) ; s_{r}=\sum_{i} \frac{a_{i}^{r}}{f^{\prime}\left(a_{i}\right)},
$$

$$
s_{r}=0, r=0,1, \ldots, n-1 ; s_{n}=1 ; s_{n+1}=\sum_{i} a_{i}=\sigma, \text { say. }
$$

We may regard (1) as $n-1$ independent linear equations in the $x_{i}^{m}$. From (2) two independent solutions are given by

$$
x_{i}^{m}=1 / f^{\prime}\left(a_{i}\right) \text { and } x_{i}^{m}=a_{i} / f^{\prime}\left(a_{i}\right) .
$$

Hence for any point $\boldsymbol{P}$ on $\Gamma$ with coordinate vector $\xi$ we have

$$
\xi_{i}^{m} f^{\prime}\left(a_{i}\right)=p+a_{i} q, \quad i=0,1, \ldots, n,
$$

for some $p, q$. If $q \neq 0$ then $x_{i}^{m}=\xi_{i}^{m}$ and $x_{i}^{m}=1 / f^{\prime}\left(a_{i}\right)$ are independent solutions of (1), while if $q=0$ then $x_{i}^{m}=\xi_{i}^{m}$ and $x_{i}^{m}=a_{i} / f^{\prime}\left(a_{i}\right)$ are. Hence, if we take the appropriate one by scalar multiplication, the coordinate vector $x$ of a generic point of $\Gamma$ satisfies

$$
x_{i}^{m}=\xi_{i}^{m}+t / f^{\prime}\left(a_{i}\right), \text { if } q \neq 0 ; \quad x_{i}^{m}=\xi_{i}^{m}+a_{i} t / f^{\prime}\left(a_{i}\right), \text { if } q=0 ;
$$

where $t$ is some indeterminate.

2.3. The points of $\Gamma$ in the face of $S$ opposite $X_{0}$ are given by $p: q=a_{0}:-1$. Since the $a_{i}$ are distinct only the first coordinate of any of these points is zero. On extracting the various $m$ th roots in (3) we obtain $m^{n-1}$ distinct points of $\Gamma$ in the face. Suppose that $P$ is one of these, so that $\xi_{0}=0$, but $\xi_{i} \neq 0$ for $i \geqq 1$. In this case (4) becomes, in view of (3),

$$
x_{0}^{m}=\frac{t}{f^{\prime}\left(a_{0}\right)} ; \quad x_{i}^{m}=\xi_{i}^{m}\left(1+\frac{t}{p+a_{i} q}\right), \quad i=1,2, \ldots, n .
$$


The extraction of $m$ th roots produces, in accordance with general theory, fractional power series in $t^{1 / m}$. So we must write $u$ for this, and then we obtain the Puiseux expansion

$$
\begin{array}{r}
x_{0}=\frac{u}{\left[f^{\prime}\left(a_{0}\right)\right]^{1 / m}} ; \quad x_{i}=\xi_{i}\left(1+\frac{K_{1} u^{m}}{p+a_{i} q}+\frac{K_{2} u^{2 m}}{\left(p+a_{i} q\right)^{2}}+\ldots+\frac{K_{n-1} u^{(n-1) m}}{\left(p+a_{i} q\right)^{n-1}}+\ldots\right), \\
i \geqq 1 ;
\end{array}
$$

of a place. Here $K_{r}$ is the coefficient of $v^{r}$ in the binomial expansion of $(1+v)^{1 / m}$ and so is non-zero. This place has centre $P$ and so is the place at $P$. The other solutions of (5) give the places at the other points of $\Gamma$ in the face $x_{0}=0$.

Let $A$ be the $n \times n$ matrix whose $i$ th row is, for $i=1,2, \ldots, n$,

$$
\xi_{i}, K_{1} \xi_{i}\left(p+a_{i} q\right)^{-1}, K_{2} \xi_{i}\left(p+a_{i} q\right)^{-2}, \ldots, K_{n-1} \xi_{i}\left(p+a_{i} q\right)^{-(n-1)} .
$$

If we remove a factor $\xi_{i}$ from the $i$ th row and $K_{j-1}$ from the $j$ th column for all relevant $i$ and $j$, then we see that the determinant of $A$ is a non-zero multiple of the Vandermonde determinant in the $\left(p+a_{i} q\right)^{-1}$. These are distinct since $q \neq 0$ and the $a_{i}$ are, so the Vandermonde determinant is non-zero and $A$ is nonsingular. Let $B$ be the $n \times n$ matrix whose $i$ th row is, for $i=1,2,3, \ldots, n$,

$$
\left(p+a_{1} q\right)^{i-2} \xi_{1}^{m-1},\left(p+a_{2} q\right)^{i-2} \xi_{2}^{m-1}, \ldots,\left(p+a_{n} q\right)^{i-2} \xi_{n}^{m-1} .
$$

$B$ is nonsingular: its determinant is a non-zero multiple of the non-zero Vandermonde determinant in the distinct $p+a_{i} q$. If $i>j$ then the $(i j)$ th entry of $B A$ is, to within a factor $K_{j-1}$ if $j>1$,

$$
\sum_{l=1}^{n}\left(p+a_{l} q\right)^{i-j-1} \xi_{l}^{m}=\sum_{l=1}^{n} \frac{\left(p+a_{l} q\right)^{i-j}}{f^{\prime}\left(a_{l}\right)}=\sum_{l} \frac{\left(p+a_{l} q\right)^{i-j}}{f^{\prime}\left(a_{l}\right)}=0,
$$

where we have used in succession (3), the fact that $p+a_{0} q=0, \operatorname{rand}$ (2) as $n-1 \geqq i-j \geqq 1$. So $B A$ is upper-triangular; let $L_{i}$ denote its non-zero $i$ th diagonal entry.

In the coordinate system given by

$$
y_{0}=\left[f^{\prime}\left(a_{0}\right)\right]^{1 / m} x_{0},\left(y_{1}, y_{2}, \ldots, y_{n}\right)^{\prime}=B\left(x_{1}, x_{2}, \ldots, x ;\right.
$$

the equations of our place become

$$
y_{0}=u ; y_{i}=L_{i} u^{(i-1) m}+\text { higher powers of } u, i \geqq 1 .
$$

Apart from an interchange of the suffices 0,1 this is the canonical form of Section 1. Hence the osculating $[s]$ at $P$ has $m s$-point contact, and is given by $y_{s+1}=y_{s+2}=\ldots=y_{n}=0$. Thus in the original coordinates the osculating $[s]$ is given by

$$
\sum_{l}\left(p+a_{l} q\right)^{i-2} \xi_{l}^{m-1} x_{l}=0, \quad i=s+1, \ldots, n
$$

we have again used the fact that $\xi_{0}=0$. A similar argument gives the same equations for the osculating [s] at a point of $\Gamma$ in any face of $S$, and proves that such an $[s]$ has $m s$-point contact. 
We may quickly explain the motivation for the construction of this $B$. To obtain the place in canonical form we need a $B$ so that $B A$ is upper-triangular, and to obtain simple equations for the osculating [s] we need a simple such $B$. Having already encountered Vandermonde matrices, and knowing that it is the $m$ th powers of $\xi_{i}$ that can be expeditiously handled in conjunction with $p+a_{i} q$, one is compelled to seek a $B$ whose $i$ th row is, for some $h$ independent of $i$,

$$
\left(p+a_{1} q\right)^{i+h} \xi_{1}^{m-1},\left(p+a_{2} q\right)^{i+h} \xi_{2}^{m-1}, \ldots,\left(p+a_{n} q\right)^{i+h} \xi_{n}^{m-1} .
$$

Use of (2) forces one to take $h=-2$.

2.4. Suppose, now, that $P$ is in no face of $S$, so $\xi_{i} \neq 0$ for all $i$. We apply the techniques of Section 2.3; the detail now runs more smoothly. We suppose, first, that $q \neq 0$. Then from (3) and (4) we have

$$
x_{i}^{m}=\xi_{i}^{m}\left(1+t /\left(p+a_{i} q\right)\right), \quad i=0,1,2, \ldots, n .
$$

Hence, the place at $P$ is given by

$$
x_{i}=\xi_{i}\left(1+\frac{K_{1} t}{p+a_{i} q}+\frac{K_{2} t^{2}}{\left(p+a_{i} q\right)^{2}}+\ldots+\frac{K_{n} t^{n}}{\left(p+a_{i} q\right)^{n}}+\ldots\right), \quad i=0,1,2, \ldots, n .
$$

If, next, $q=0$, then the second alternative of (4) must be used with (3), and we find, now, that the place at $P$ is given by

$$
x_{i}=\xi_{i}\left(1+\frac{K_{1} a_{i} t}{p}+\frac{K_{2} a_{i}^{2} t^{2}}{p^{2}}+\ldots+\frac{K_{n} a_{i}^{n} i^{n}}{p^{n}}+\ldots\right), i=0,1,2, \ldots, n .
$$

In either case let $C$ be the $(n+1) \times(n+1)$ matrix whose $(i+1)$ th row, $i=0,1, \ldots, n$, has for its entries the coefficients, in order, of $1, t, t^{2}, \ldots, t^{n}$ in the expansion of $x_{i}$. Motivated as in Section 2.3 we take $D$ to be the $(n+1) \times(n+1)$ matrix whose $(i+1)$ th row, for $i=0,1, \ldots, n$, is

and

$$
\left(p+a_{0} q\right)^{i-2} \xi_{0}^{m-1},\left(p+a_{1} q\right)^{i-2} \xi_{1}^{m-1}, \ldots,\left(p+a_{n} q\right)^{i-2} \xi_{n}^{m-1}, \quad \text { if } q \neq 0,
$$

$$
a_{0}^{n-i} \xi_{0}^{m-1}, a_{1}^{n-i} \xi_{1}^{m-1}, \ldots, a_{n}^{n-i} \xi_{n}^{m-1}, \quad \text { if } q=0 .
$$

If the weaker form of the canonical form (1) of $\Gamma$ is used, so that some $a_{i}$ may be zero then, if $q=0, a_{i}^{0}$ is here taken to be 1 . Arguments similar to those used for $A$ and $B$ in Section 2.3 show that $C$ and $D$ are nonsingular. If $0 \leqq i, j \leqq n$ and $i>j$, then if $q \neq 0$ the $(i+1, j+1)$ th entry of $D C$ is, to within a factor $K_{j}$ if $j \geqq 1$,

$$
\sum_{l}\left(p+a_{l} q\right)^{i-j-2} \xi_{l}^{m}=\sum_{l} \frac{\left(p+a_{l} q\right)^{i-j-1}}{f^{\prime}\left(a_{l}\right)}=0
$$

by (3) and (2) since $0 \leqq i-j-1<n$; while if $q=0$ the entry is, to within the non-zero factor $K_{j} p^{-j}$ if $j \geqq 1$,

$$
\sum_{l} a_{l}^{n-i+j} \xi_{l}^{m}=p \sum_{l} \frac{a_{l}^{n-i+j}}{f^{\prime}\left(a_{l}\right)}=0
$$


by (3) and (2) since $0 \leqq n-i+j<n$. Thus $D C$ is upper-triangular; let $M_{i}$ be its non-zero $(i+1)$ th diagonal entry. In the coordinate system given by

$$
\left(y_{0}, y_{1}, \ldots, y_{n}\right)^{\prime}=D\left(x_{0}, x_{1}, \ldots, x_{n}\right)^{\prime}
$$

the place at $P$ is given by

$$
y_{i}=M_{i} t^{i}+\text { higher powers, } i=0,1, \ldots, n \text {. }
$$

Thus the osculating $[s]$ at $P$ is ordinary and is given by

Hence it is given by

$$
y_{s+1}=y_{s+2}=\ldots=y_{n}=\mathbf{0} \text {. }
$$

and

$$
\sum\left(p+a_{l} q\right)^{i-2} \xi_{l}^{m-1} x_{l}=0, \quad i=s+1, \ldots, n, \quad \text { if } q \neq 0,
$$

$$
\sum_{l} a_{l}^{n-i} \xi_{l}^{m-1} x_{l}=0, \quad i=s+1, \ldots, n, \quad \text { if } q=0 .
$$

2.5. Before eliminating $p$ and $q$ from (6) and (7) we summarise the results for the hyperosculating spaces in

Theorem 1. The points of hyperosculation of $\Gamma$ are its $(n+1) m^{n-1}$ intersections with the faces of $S$. At such a point the osculating [s] has ms-point contact. All the other points on $\Gamma$ are ordinary.

\section{The equations of the osculating spaces}

3.1. It is immediate from (6), (7) and (8) that the equation of the osculating prime to $\Gamma$ at any point $\xi$ is

$$
\sum_{l}\left(p+a_{l} q\right)^{n-2} \xi_{l}^{m-1} x_{l}=0 .
$$

From (2) and (3) we have

so we obtain

$$
\sum_{i} a_{i}^{n-1} \xi_{i}^{m}=q ; \quad \sum_{i} a_{i}^{n} \xi_{i}^{m}=p+\sigma q ;
$$

Theorem 2. The equation of the osculating prime at $\xi$ on $\Gamma$ is

$$
\sum_{i}\left\{\sum_{i} a_{i}^{n} \xi_{i}^{m}-\left(\sigma-a_{l}\right) \sum_{i} a_{i}^{n-1} \xi_{i}^{m}\right\}^{n-2} \xi_{i}^{m-1} x_{l}=0 .
$$

This equation, for an ordinary point $\xi$, was given in (2, p. 336). An alternative expression is found by substituting (3) in (9) to obtain for the equation of the osculating prime

$$
\sum_{l}\left\{f^{\prime}\left(a_{l}\right)\right\}^{n-2} \xi_{l}^{m(n-1)-1} x_{l}=0 .
$$

3.2. From (6), (7) and (8) we also obtain

Theorem 3. If $s<n-1$ then the osculating $[s]$ at $\xi$ on $\Gamma$ is given by

$$
\sum_{i}\left\{\sum_{i} a_{i}^{n} \xi_{i}^{m}-\left(\sigma-a_{l}\right) \sum_{i} a_{i}^{n-1} \xi_{i}^{m}\right\}^{i-2} \xi_{l}^{m-1} x_{l}=0, \quad i=s+1, \ldots, n
$$


EXCEPT for the points where $\xi_{i}^{m}=\left[f^{\prime}\left(a_{i}\right)\right]^{-1}$ for all $i$, when it is given by

$$
\sum_{l} a_{l}^{n-i} \xi_{l}^{m-1} x_{l}=0, \quad i=s+1, \ldots, n .
$$

At a point where $\bar{\zeta}_{i}^{m}=\left[f^{\prime}\left(a_{i}\right)\right]^{-1}$ for all $i$, each equation of the first set is that of the osculating prime: this is perhaps most clearly seen by putting $q=0$ in (7). Symmetry is, however, restored if we consider the Grassmann coordinates of the $[s]$. Elementary determinant theory gives from the above equations, or perhaps more obviously from (6), (7) and (8) directly,

Theorem 4. The dual Grassmann coordinates of the osculating [s] to $\Gamma$ at any point $\xi$ are given by

$$
\pi_{i j \ldots}=\left[f^{\prime}\left(a_{i}\right) f^{\prime}\left(a_{j}\right) \ldots\right]^{s-1}\left[\xi_{i} \xi_{j} \ldots\right]^{m s-1} V_{i j \ldots},
$$

where $V_{i j \ldots}$ is the $(n-s) \times(n-s)$ Vandermonde determinant in $a_{i}, a_{j}, \ldots$

In (2, pp. 336, 337) the dual coordinates of the osculating planes and solids to a curve of intersection of $n-1$ general primals were given, but, again, these are only valid at the ordinary points of $\Gamma$.

\section{The group of $\Gamma$}

4.1. It follows from Theorem 3 that the tangent lines to $\Gamma$ at its points in one face of $S$ concur at the opposite vertex, and are the only tangents to $\Gamma$ through this vertex. Further, the tangents at two points of hyperosculation in distinct faces of $S$ do not meet. We may prove this as follows. A point $P$ of $\Gamma$ in the face $x_{i}=0$ may be taken as $\xi$ with $\xi_{l}^{m}=\left(a_{l}-a_{i}\right) / f^{\prime}\left(a_{l}\right)$ for all $l$, and a point $Q$ in $x_{j}=0(i \neq j)$ as $\eta$ with $\eta_{l}^{m}=\left(a_{l}-a_{j}\right) / f^{\prime}\left(a_{l}\right)$, by (3). Were the tangents $P X_{i}, Q X_{j}$ to meet then $P Q$ would meet $X_{i} X_{j}$. Hence there would exist $\lambda, \mu$, not both 0 , such that $\lambda \xi_{l}-\mu \eta_{l}=0$ for all $l \neq i, j$, and consequently

$$
\lambda^{m}\left(a_{l}-a_{i}\right)=\mu^{m}\left(a_{l}-a_{j}\right) \text { for all } l \neq i, j .
$$

Since $n \geqq 3$ and the $a_{l}$ are distinct this is impossible.

The group of projectivities fixing $\Gamma$ permutes the points of hyperosculation. Any of its elements takes one pair of points with concurrent tangents to another. Hence the group fixes $S$, and has a normal subgroup, $G$ say, fixing each vertex of $S$. The existence of particular relationships among the $a_{l}$ will imply that the full group of $\Gamma$ is bigger than $G$.

Suppose that an element of $G$ has for a matrix, which must be diagonal, $\operatorname{diag}\left(\alpha_{0}, \alpha_{1}, \ldots, \alpha_{n}\right)$. This takes the point $P$ in $x_{i}=0$ considered above to the point on $\Gamma$ with coordinates $\left(\alpha_{0} \xi_{0}, \alpha_{1} \xi_{1}, \ldots, \alpha_{n} \xi_{n}\right)$. Since all points of $\Gamma$ in $x_{i}=0$ are given by $\mathrm{p}: q=-a_{i}: 1$ we see from (3) that there is a non-zero $\lambda$ so that $\alpha_{l}^{m} \xi_{l}^{m}=\lambda \xi_{l}^{m}$ for all $l$. Since $\xi_{i}$ alone of the $\xi_{l}$ is zero we see that the $\alpha_{l}^{m}$ for $l \neq i$ are all equal. Since $i$ can vary we conclude that the $\alpha_{l}^{m}$ are all equal, and hence that an element of $G$ has for a matrix diag $\left(\lambda_{0}, \lambda_{1}, \ldots, \lambda_{n}\right)$, where each $\lambda_{1}$ is an $m$ th root of unity. Conversely, such a matrix gives a projectivity fixing each of the defining primals (1) of $\Gamma$ and so in $G$. There are $m^{n+1}$ such com- 
muting matrices, of which $m$ are scalar. Hence $G$ is an abelian group of order $m^{n}$ and exponent $\mathrm{m}$. It is clear from (1) that these matrices do give a subgroup of the group of $\Gamma$, but it is not obvious ab initio that any projectivity fixing $\Gamma$ and each vertex of $S$ must fix each of the defining $m$-ics.

From (3) and the matrix form above we see that the orbit of a point of $\Gamma$ under $G$ consists of those points with the same $p: q$. Hence, on extraction of $m$ th roots in (3), we see that the orbits have sizes $m^{n}$ and $m^{n-1}$ for ordinary and hyperosculation points respectively. Hence the points of hyperosculation are those points of $\Gamma$ which have non-trivial stabilisers in $G$.

4.2. Let $P$ be a point of hyperosculation of $\Gamma$ in the face $x_{i}=0$ of $S$. Since $P$ lies in no $[n-2]$ spanned by vertices of $S$, each element of the stabiliser in $G$ of $P$ must, by the fundamental theorem of projective geometry, fix the face $x_{i}=0$ pointwise. Hence the stabiliser consists of those homologies in $G$ with vertex $X_{i}$ and axis $x_{i}=0$, together with the identity. Any such homology has a matrix of the form diag $\left(1,1, \ldots, 1, \lambda_{i}, 1,1, \ldots, 1\right)$ where only the $(i+1)$ th diagonal entry is not 1. By Section $4.1 \lambda_{i}$ must be an $m$ th root of unity. A particular such homology is $h_{i}$ given by $\lambda_{i}=\rho$, where $\rho$ is a primitive $m$ th root of 1 . The stabiliser of $P$ is $\left\langle h_{i}\right\rangle$, a cyclic group of order $m$. Thus the stabiliser of a point of hyperosculation of $\Gamma$ is a cyclic group of order $m$ consisting, apart from the identity, of homologies.

From the matrix form of $G$ we see that $G=\left\langle h_{0}, h_{1}, \ldots, h_{n}\right\rangle$. The image under $h_{i}$ of any point lies on the join of that point to $X_{i}$. Further the orbit under $\left\langle h_{i}\right\rangle$ of any point not at $X_{i}$ nor in $x_{i}=0$ has size $m$. Hence the points of hyperosculation in a face of $S$ lie by $m$ 's on lines through each vertex of $S$ in the face. Another consequence is that all members of the orbit of a subspace $L$ under the group generated by some of the $h_{i}$ lie in the join of $L$ to the vertices of these homologies.

The tangent to $\Gamma$ at $\xi$ meets the $[n-2]: x_{n-1}=x_{n}=0$ if and only if its dual Grassmann coordinates satisfy $\pi_{012} \cdots_{n-2}=0$. By Theorem 4 this is impossible at an ordinary point. Take, then, an ordinary point $P^{\prime}$ on $\Gamma$. Its tangent line $t$ to $\Gamma$ does not meet the $[n-3]$ spanned by $X_{0}, X_{1}, \ldots, X_{n-3}$. The action of $\left\langle h_{0}, h_{1}, \ldots, h_{n-3}\right\rangle$ gives an orbit of $m^{n-2}$ tangents to $\Gamma$ lying in the prime joining $t$ to the $[n-3]$ spanned by $X_{0}, X_{1}, \ldots, X_{n-3}$, and whose distinct contacts lie in the join of $P^{\prime}$ to the $[n-3]$. For the $m$ and $n$ under consideration $m^{n-2} \geqq n$ except when $m=2$ and $n=3$. Hence, apart from the elliptic quartic in [3], $\Gamma$ has an infinity of n-ritangent primes. A general curve in $[n]$ has only a finite number of such primes; their number is given by a formula of Castelnuovo (see 1, Vol. 6, p. 45). In fact, apart from the quartic, $\Gamma$ has $\left(\begin{array}{c}n+1 \\ 3\end{array}\right)$ families of $n$-ritangent primes, one of each family joining $t$ to an $[n-3]$ spanned by $n-2$ vertices of $S$.

If $n \geqq 4$, and we consider the action of $\left\langle h_{0}, h_{1}, \ldots h_{n-4}\right\rangle$ on the osculating plane at $P^{\prime}$, then we see that there is an infinity of primes having 3-point contact at $m^{n-3}$ distinct points. And so on. 


\section{REFERENCES}

(1) H. F. BAKER, Principles of Geometry, Vols. 5, 6 (Cambridge, 1933).

(2) R. H. Dye, Osculating primes to curves of intersection in 4-space, and to certain curves in $n$-space, Proc. Edinburgh Math. Soc. (II) 18 (1973), 325-338.

(3) J. G. Semple and G. T. KneEbone, Algebraic Curves (Oxford, 1959).

SCHOOL OF MATHEMATICS

UNIVERSITY OF NEWCASTLE UPON TYNE 